\title{
Q-ROBOT CAST FOR LADLE AREA: REACHING NEW LEVELS OF AUTOMATION AND SAFETY IN CONTINUOUS CASTING *
}

\author{
Paolo Demetlika \\ Alessandro Ardesi \\ Ingrid Visentini ${ }^{3}$ \\ Manuel Martin ${ }^{4}$ \\ Luca Cestari 5 \\ Daniele Comand ${ }^{6}$
}

\begin{abstract}
In this paper it is shown how the "Zero Operator" concept on the casting floor has been reached by adoption of a standard 6 axis industrial anthropomorphic robot coupled with the most innovative machine vision technology. This has been achieved by automating all the operations related to the ladle and tundish activities, including shroud manipulation, powder addition, ladle nozzle identification, nozzle opening by lancing, temperature measurement and chemical sampling.

Q-Robot CAST family products are the result of the successful alliance between industrial robotics and the most advanced technology in process automation. This led, for each production area, to the introduction of flexible automation in dangerous places, even where some degree of human intervention is still necessary. By using anthropomorphic robots, human operators can be reassigned to safer environments. Furthermore, robots are suited to deal with repetitive and labor intensive tasks thus guaranteeing a high process quality.

Keywords: Robotics in steelmaking - continuous casting, industrial anthropomorphic 6 axis robot, safety, robotic vision system, vision-guided robotics; "Zero Operators" concept.
\end{abstract}

1 Danieli Automation S.p.A., Via Bonaldo Stringher 4 - 33042 Buttrio (UD) - Italy Phone: +390432 518502, Fax: +390432 518125, p.demetlika@dca.it.

Danieli Automation S.p.A., Via Bonaldo Stringher 4 - 33042 Buttrio (UD) - Italy

Danieli Automation S.p.A., Via Bonaldo Stringher 4 - 33042 Buttrio (UD) - Italy

Danieli Automation S.p.A., Via Bonaldo Stringher 4 - 33042 Buttrio (UD) - Italy

Danieli \& C. Officine Meccaniche. S.p.A., via Nazionale, 41 - 33042 Buttrio (UD) - Italy

Danieli \& C. Officine Meccaniche. S.p.A., via Nazionale, 41 - 33042 Buttrio (UD) - Italy 


\section{INTRODUCTION}

There is a strong momentum surrounding the adoption of robotic technology within the steelmaking plants in order to cope with the always-increasing need for safety improvements. In particular, this trend can reduce the risk for human operators, and make repetitive operations faster and more efficient and reliable. The main safety issue in the continuous casting process is related to the human presence in the ladle area.

Based on "Zero Operators" concept, where no men are physically involved near the caster, Danieli Automation developed Q - Robot CAST Ladle area. It is a complex multifunctional machine, where all the advantages of a 6-axis anthropomorphic industrial robot have been exploited. The main activities are carried out in fully automatic mode, supported by robotic technology and other forms of automation (e.g. vision system for identifying the ladle nozzle position, quick-change coupling system, and automated auxiliary devices, etc.). Moreover, all the system functionalities can be carried out also in manual mode from the remote control station, where the robot can be guided by a human operator with a joystick control device in order to guarantee system availability.

This approach eases the widespread adoption of robotics which guarantees more operating speed, more efficiency and more productivity, making this technology attractive thanks to a short return on investment.

\section{Q-ROBOT CAST}

\subsection{System operation}

The Q - Robot CAST Ladle is a multifunctional robotic system that performs automatically the following actions in the ladle area:

- $\quad$ ladle nozzle position identification;

- $\quad$ shroud handling with the support of an auxiliary manipulator;

- $\quad$ nozzle opening by lancing;

- $\quad$ tundish powder management;

- $\quad$ tundish temperature measurements and chemical sampling analysis.

There are also few additional auxiliary tasks that the robot can perform in order to increase the automation and safety level as well as the efficiency of the process. An example is the shroud cleaning phase which is required when shroud clogging occurs. The shroud is positioned on the cleaning station where oxygen is being blown in complete safety and where no operators are present.

In the following chapters, each task will be described separately.

\subsection{Ladle nozzle identification and shroud manipulation}

The flow of liquid steel from ladle to tundish is controlled with the opening of a sliding gate valve. Protection from air re-oxidation of the flowing steel stream is obtained with the use of a refractory shroud that strongly reduces the generation of large-sized inclusions mainly due to oxidation of aluminum and the pickup of nitrogen; inert gas shrouding inside the refractory tube can also be used to ensure additional protection 
to the pouring steel. Insertion and removal of the shroud from the slide gate nozzle during casting can be performed by means of various devices. The typical equipment used for this task is a hydraulic or pneumatic manipulator with a movable support arm operated by casting floor personnel.

The solution developed within the Q-Robot CAST system allows performing the entire procedure in a fully automatic way, without direct involvement of human operators.

In the envisaged system, the robot is integrated with an auxiliary pneumatic manipulator for shroud handling. The positioning of all the equipment ( e.g the warehouse for robot tools and consumables) are optimized according to the specific plant layout constraints, in order to reduce cycle times for all robot operations.

A first task that the robot has to perform is the identification of the nozzle once the ladle has been settled in the working position. A stereoscopic camera is used in order to measure a precise 3D geometry of the nozzle. In order to optimize the working cycle of the robot, the vision system can be integrated on the shroud tool.

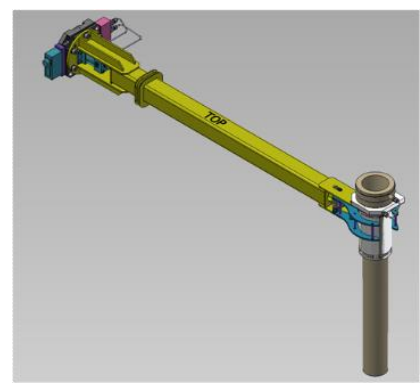

Figure 1. Shroud tool.

With the shroud tool the 6-axis robot performs two main sub-tasks:

- shroud manipulation;

- auxiliary manipulator guidance;

The shroud tool developed for this application is a long arm mounted on the robot wrist flange. At the end of the arm a pneumatic gripper has been realized for shroud manipulation and for auxiliary manipulator handling.

First of all, the robot picks the shroud from the warehouse (as shown in figure below),

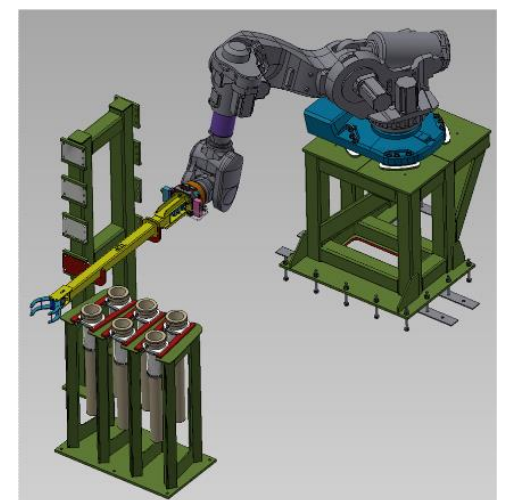

Figure 2. Shroud manipulation (left); auxiliary shroud manipulator in parking position (right). 
and mounts it on the auxiliary manipulator, which has been previously positioned in parking position (manipulator holder). Each shroud is endowed with a protective sleeve which has manifold targets: shroud protection during manipulation; quick coupling system with the robot and with the auxiliary manipulator's fork; integrated fittings for fluid distribution system (e.g. Argon). An example is shown in the figure hereunder.

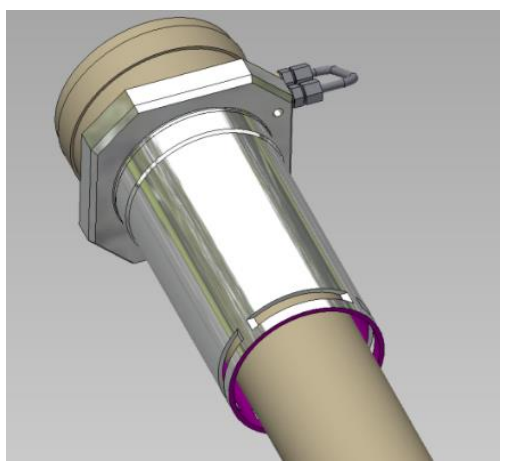

Figure 3. Shroud protective sleeve.

Once the robot has released the shroud on the manipulator's fork, it grips the manipulator arm and starts to drag it toward the final position identified previously by the vision system.

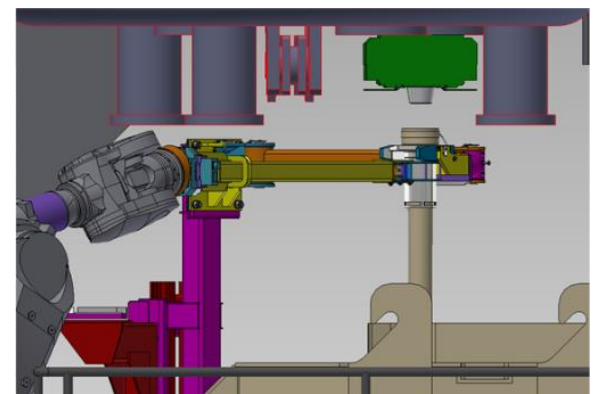

Figure 4. Robot dragging the auxiliary shroud manipulator with the shroud toward the nozzle position.

The final shroud sustainment has been assigned to the auxiliary manipulator, which exerts the required force once it has been correctly positioned under the ladle nozzle. One reached the working position under the nozzle, the robot releases the manipulator arm that starts to execute a pushing upward force attaching and maintaining the shroud fixed to the nozzle during the whole casting process.

In case of a spontaneous nozzle opening, the robot ends the task returning to the home position. Otherwise, when a non-spontaneous nozzle opening occurs, the robot cancels the running task executing a reverse path, and then starts with the "lancing" nozzle opening task.

The proposed solution with the auxiliary manipulator has many advantages, between them:

- ease of installation, there is no need for plant modifications (neither on the ladle slide valves);

- it is installed on tundish car and therefore it is mechanically independent from the ladle turret;

- in emergency cases the manipulator releases the upward force and decouples from the ladle, which becomes free to recover in safety position (facilitates the safety operations during the emergency); 
- all auxiliary connections (e.g. argon) are already integrated on-board installation

\subsection{Nozzle opening - Lancing}

When the ladle slide gate is opened successfully and the steel starts flowing without any intervention of the operator, the procedure is defined "free open“. In some cases the ladle arrives in casting position with a clogged nozzle, and the pressure exerted by the liquid steel is not sufficient to break the layer of sintered crust which prevents the flow of material. Nowadays steelmakers have optimized the operating practices in order to reduce the occurrence of ladle nozzle clogging events, with specific actions on ladle maintenance and preheating cycles, heating and stirring parameters in secondary steelmaking and with the addition in the ladle well block of chromite-based filler sands with the desired flowability and permeability characteristics.

Despite all these precautions, there is still a variable percentage of heats where ladle nozzle opening is not spontaneous and must be obtained manually by means of poking or oxygen lancing. Typically one or more consumable thermal lances are used for this task until full opening of the nozzle is achieved, and the operator involved must be very careful to handle the oxygen lance avoiding any contact with flames and steel splashes. For this purpose a specifically designed lance holder with thermal lance for oxygen blowing has been integrated in the Q-Robot CAST system. The robot picks the lance holder which has been previously prepared by the operator on the warehouse. Successively, the lance holder with the thermal lance has to be ignited. That is achieved by an electric arc ignition system where robot inserts the thermal lance between the ignition plates and moves it in order to provoke an electric arc which lights the consumable part of the lance.

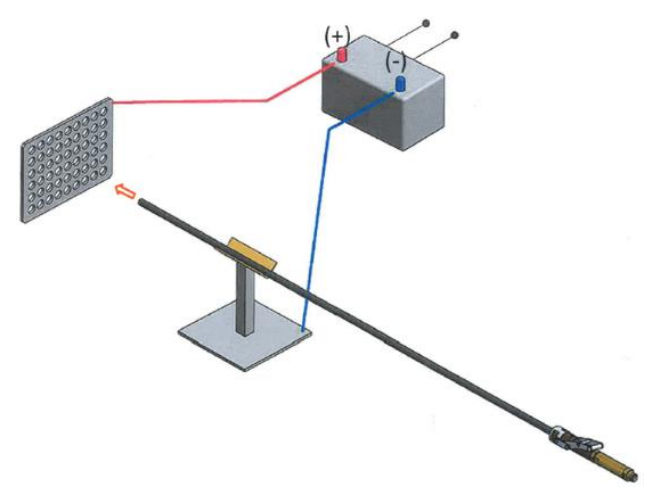

Figure 5: Electric ignition system - Courtesy of Contessi

Once the lance has been ignited, the robot moves toward the clogged nozzle, inserts the incandescent lance head inside the nozzle and starts to blow oxygen. At the same time an upside-down movement helps the nozzle opening. The procedure can be repeated as many times as required and it is finished by the operator's confirmation.

At the end, the robot releases the used lance within the warehouse and restarts with the shroud mounting procedure described in the previous chapter. In addition to the nozzle opening task, also a shroud cleaning procedure is available in case of shroud clogging. In this case, the robot drags the auxiliary manipulator and the clogged shroud toward the cleaning station, where a cleaning process by oxygen blowing is 
performed. Through a remote vision system the operator can check the shroud status and decide when the shroud cleaning task has to be terminated.

\subsection{Tundish powder management}

Tundish powder performs many important functions in continuous casting [1], [2] (e.g. ensure temperature control with the minimization of heat losses, protect the liquid steel from ambient air re-oxidation and increase the removal of floating inclusions). Maintaining a proper thickness for the layer of powder above the steel level is crucial to guarantee the best casting conditions, and this target can be achieved better with an automated powder feeding system.

For tundish powder management a properly designed canister tool is used which is filled with the required casting powder. In order to optimize the time cycle during the powder addition, two or more canister tools can be employed.

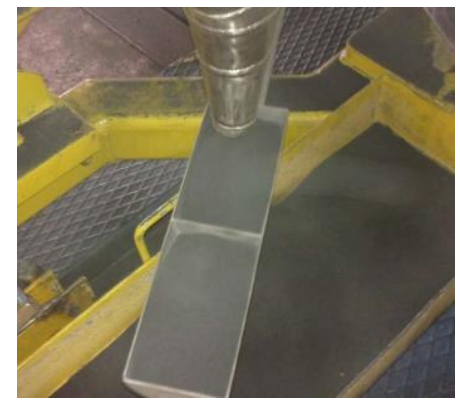

Figure 6: Canister tool for casting powder

Each canister tool is endowed with the quick change coupling system which allows the robot to cyclically distribute the casting powder. The used canister tools are stored directly under each bulk hopper and are refilled during the cycle of powder distribution performed by the robot, optimizing thus the refilling time of other canisters. The addition of the required powder quantity within each canister tool is controlled by pneumatic valves.

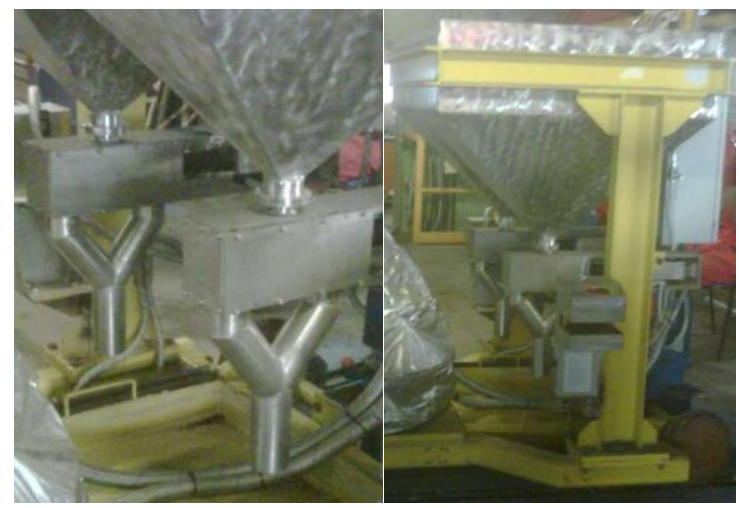

Figure 7: Powder distribution system (left);Bulk Hopper for each casting powder type (right);

Moreover, all the flexibility of a 6 axis industrial robot is exploited for a uniform powder distribution within the tundish liquid steel surface.

If different typologies of casting powder are being used, for each kind of powder there is a dedicated bulk hopper. This architecture/operating modality 
consent to achieve quick time responses for powder addition, correct control of the powder quantity as required from the process, improves the powder management (quantity for each bulk hopper i.e. transportation and storage inside the plant) as well as the process quality since different type of powder are employed and managed. In addition to the proposed solution, the casting powder can be managed also with bag packaging. In this case, dependently also on the bag storage, different tools for bag picking can be used (e.g. vacuum tool, clam shell mechanical gripper, fork gripper, bag gripper).

\subsection{Tundish temperature measurements and chemical sampling analysis}

Accurate and fast monitoring of steel chemistry and temperature changes in tundish is critical to the quality of cast products. This task is accomplished by means of disposable sampling lances which are usually handled by the operator on the casting floor. In order to guarantee reliability and repeatability of the measure and to ensure a safe operation, the lance must be properly submerged into the steel bath.

The multifunction robot presented in this paper is also equipped with a tundish measuring and sampling system (i.e. system for temperature measurements and chemical composition analysis of the steel bath). This system is composed of a control unit and a dedicated sampling lance which is handled by the robot.

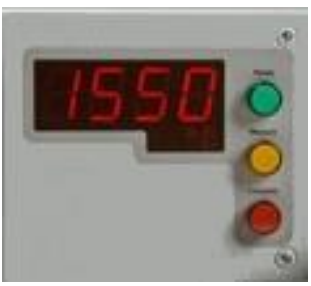

Figure 8: Control and temperature measuring unit

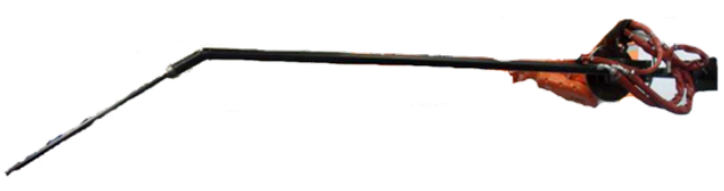

Figure 9: Temperature measuring and sampling lance

A water cooling system is used to achieve a great durability of the measuring lance. Different measuring probes can be used and managed by automatic loading and unloading dispensers that are installed within the robot working area. The storage capacity can vary accordingly to the process requirements. This greatly reduces the manual operator's intervention, limiting any kind of injuries and reduces the process sampling time. 


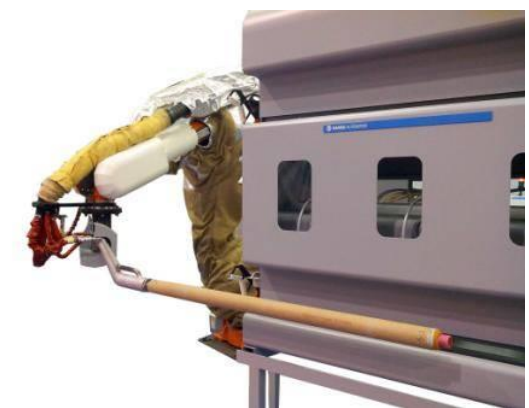

Figure 10: Automatic cartridge probe charging

The high reliability of the sampling operation is achieved by maintaining a constant depth and tilt angle of the lance, which can be settled from the HMI. Once the measurement has been done, the robot releases the used probe on the cartridge extractor and turn on ready position for the next temperature measuring or sampling cycle.

\subsection{System Architecture}

In this chapter a brief description of the system architecture and various components of the Q-Robot CAST Ladle are given. In the following figure a schematic system architecture and communication interactions between the components are shown.

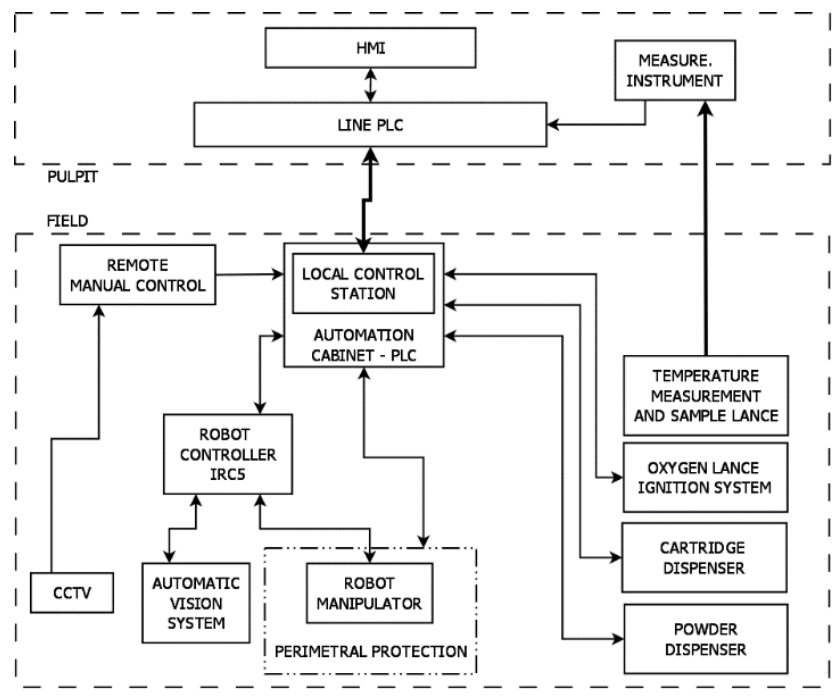

Figure 11: $\bar{Q}-\bar{R}$ obot $\bar{C} A \bar{S} \bar{T}$ Ladle system architecture

\subsection{Robot and controller unit}

The main component of the Q-Robot CAST Ladle system is an anthropomorphic robot with its own controller as shown in the figure below. 


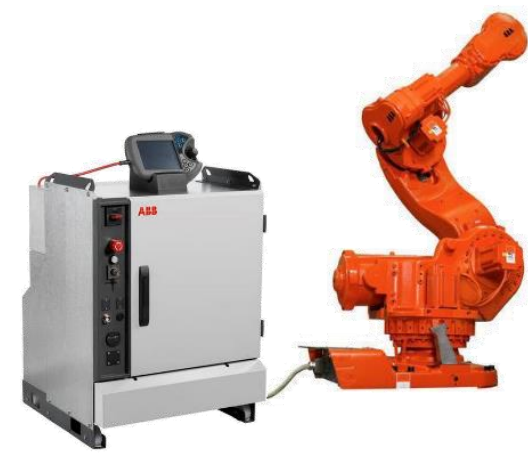

Figure 12: Industrial 6 axis robot with controller

The manipulator is an industrial kinematic system with six axes and six degrees of freedom. It must be installed close to the working area in the plant in order to guarantee a straightforward approach. The main scope of the robot is the handling of the different tools used for each task. The manipulator represents the state-of-the-art of industrial robots with the highest technology available and has been specifically designed to satisfy the needs of industrial processes. It can be used in harsh environments, and can work with a $100 \%$ continuous duty cycle. Furthermore, the robot is equipped with a particular high temperature resistant and protective suite. The manipulator combines excellent movement and control capabilities having compact arm and wrist dimensions. The compact design ensures easy installation of the manipulator. The sturdy construction contributes to high reliability and long intervals between maintenance.

\subsection{Quick-change coupling system}

Each task performed by robot requires different tool. With the aim to optimize the onboard installation and to reduce the cycle time for each operation, a special quickchange coupling system has been chosen and adopted. Generally, it is constituted by the following components:

- master side -installed on the robot wrist flange;

- slave side -mounted on each tool that has to be used and manipulated by the robot;

- auxiliary devices -valves, distribution blocks, sensors and electrical connectors can be integrated in the system;

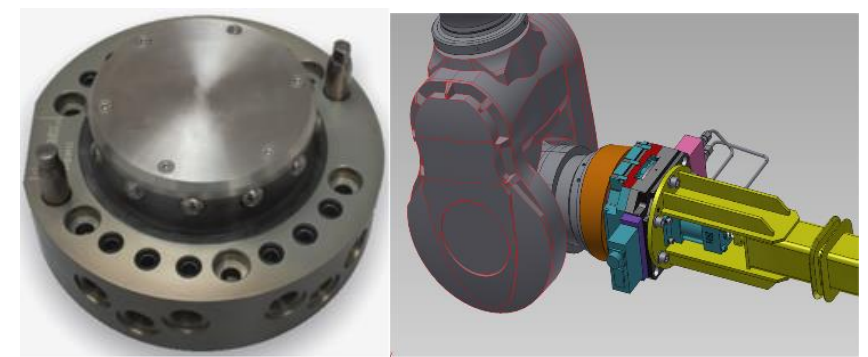

Figure 13: Quick-change coupling system - Master side(left)-Courtesy of RoboTools; Quick-change coupling system assembly with end-effector (right);

The designed quick-change coupling system performs two main activities: mechanical coupling -to mechanically interchange and use different tools; 
feed through elements -to feed through the quick-change coupling system all the electrical and fluid connections required for the process.

\subsection{Vision system}

The Q-Robot CAST Ladle is equipped with a vision system that allows identifying the geometrical position of the ladle nozzle. It is composed by different optical sensors (e.g. industrial cameras) directly connected to the industrial PC which executes the vision algorithms. The machine vision system directly interacts with the robot control unit, and provides the required positioning information.

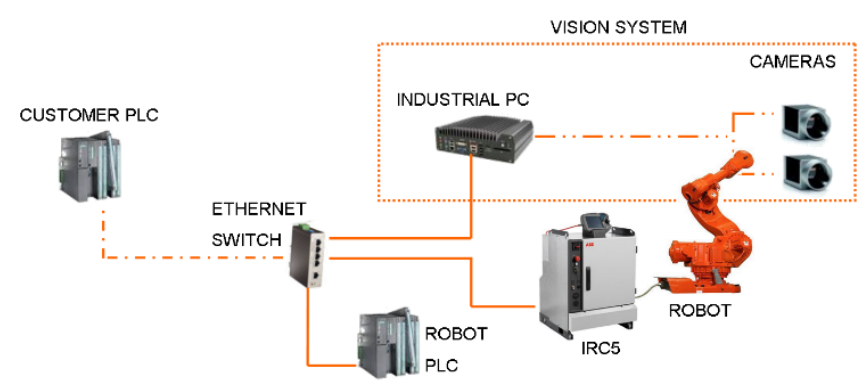

Figure 14: Vision system architecture and communication network

In the present case, it is based on an off-the-shelf stereo camera. The point cloud collected by the camera sensor is segmented and processed to extract with maximum accuracy the target position. The "eye-in-hand" architecture has been adopted where a stereo camera with integrated illuminator and pattern projector is mounted on the robot end-effector. For the thermal and mechanical protection a special enclosure has been designed. An example of the vision system on the robot arm is shown in the following figure during laboratory test installation.

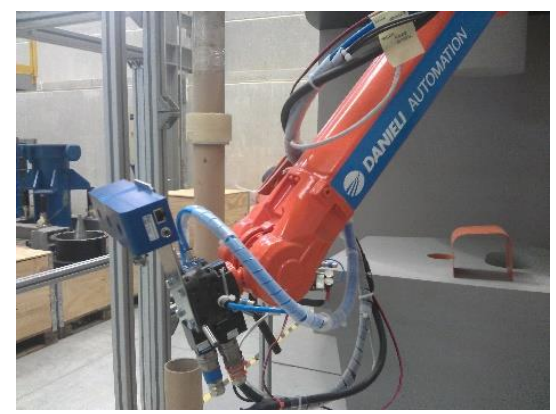

Figure 15: Eye-in-hand vision system

This vision system architecture guarantees good accuracy and the ability to explore the workspace achieving thus higher system flexibility [3]. Indeed, it can precisely measure the distance from the vision system frame to a target and thus allowing the identification of the nozzle. In order to map the vision system measurements to the robot workspace frame a calibration process is required. In this way the robot can direct its end-effector toward the identified nozzle. For the calibration process a dedicated calibration plate is used. The calibration process also allows the robot to precisely move the sensor for inspecting complex 3D parts and thus to reconstruct a 3D scenes with a moving camera [4]. In the following figures different views of a mockup nozzle are shown. 

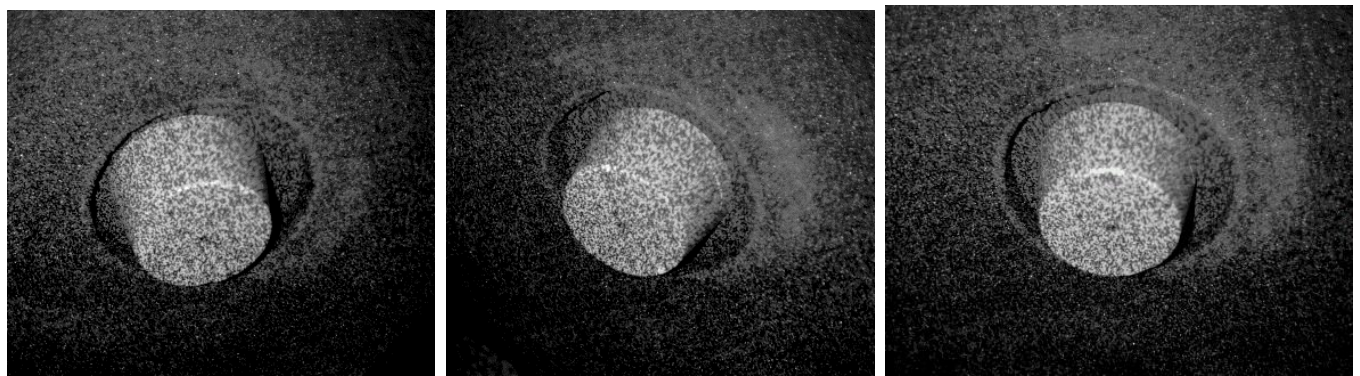

Figure 16: Camera views from different angles of a mockup nozzle

In the figure hereunder a reconstruction and identification of the central point of the ladle nozzle mockup is shown.

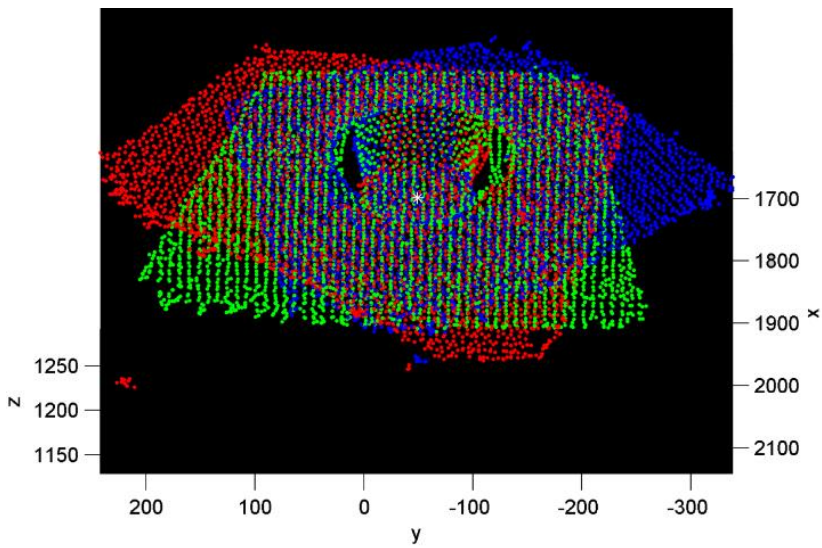

Figure 17: Nozzle mockup 3D geometry and position reconstruction

\section{CONCLUSION}

The Q-Robot CAST Ladle presented in this paper shows how the adoption of the robotics technology within the steelworks combined with other advanced automation technologies brings twofold benefits. In particular it can be summarized as follows:

- improvement of the safety level since no operators are physically involved in dangerous activities in the casting floor area (i.e. reduced operators' presence in hazardous area, minimization of operators involvement in recurrent time consuming and risky tasks, low exposure to high temperatures, high noise and pollution levels);

- the achieved flexibility brings the entire process to higher automation levels improving the process efficiency.

\section{Acknowledgments}

Abbreviations:

$\mathrm{HMI}$ - Human machine interface;

PC - Personal computer;

\section{REFERENCES}

1 Fruehan, J.R.: The Making, Shaping and Treating of Steel; 11th ed.,The AISE Steel Foundation, Pittsburgh 1998

2 Irving, W.R.: Continuous Casting of Steel; The Institute of Materials, 1993 
3 Horaud, R.; Dornaika, F.: Hand-Eye Calibration; Int. Journal of Robotics Research, Vol. 14, No. 3, P. 195-210, June 1995

4 Hartley, R.; Zisserman, A.: Multiple View Geometry in Computer Vision; Cambridge University Press, 2000. 